\title{
CORRECTION
}

\section{Correction to: Foreword to the memorial issue for Professor Roberto Marassi}

\author{
Pawel J. Kulesza ${ }^{1} \cdot$ Francesco Nobili $^{2} \cdot$ Sonia Dsoke ${ }^{3} \cdot$ Vito Di Noto $^{4} \cdot$ Iwona A. Rutkowska ${ }^{1}$
}

Published online: 6 January 2022

○) Springer-Verlag GmbH Germany, part of Springer Nature 2022

\section{Correction to: Journal of Solid State Electrochemistry https://doi.org/10.1007/s10008-021-05099-2}

The original article contained a mistake.

Author name "habil Iwona A. Rutkowska" should be corrected to "Iwona A. Rutkowska" and is now correctly displayed above.

The original article has been corrected.

Publisher's Note Springer Nature remains neutral with regard to jurisdictional claims in published maps and institutional affiliations.

The original article can be found online at https://doi.org/10.1007/ s10008-021-05099-2.

Pawel J. Kulesza

pkulesza@chem.uw.edu.pl

Francesco Nobili

francesco.nobili@unicam.it

Sonia Dsoke

sonia.dsoke@kit.edu

Vito Di Noto

vito.dinoto@unipd.it

Iwona A. Rutkowska

ilinek@chem.uw.edu.pl

1 Faculty of Chemistry, University of Warsaw, Pasteura 1, 02093 Warsaw, Poland

2 School of Science and Technology - Chemistry Division, University of Camerino, Via S. Agostino, 1, 62032 Camerino, MC, Italy

3 Karlsruher Institut Für Technologie, Campus Nord, HIU, Eggenstein-Leopoldshafen, Baden-Wurttemberg, Germany

4 Department of Chemical Sciences, University of Padova, Via Marzolo 1, I-35131 Padova, Italy 